\title{
CYCLES ÉVANESCENTS \\ (EN LIEN AVEC LA THÉORIE AUTOMORPHE)
}

\author{
par
}

Henri Carayol

Table des matières

1. Introduction.................................. 1

2. Le cas des courbes............................ 2

3. Le cas de la dimension supérieure................. 12

Références.................................... 23

\section{Introduction}

L'objet de cet article est d'expliquer le lien entre la théorie automorphe et celle des cycles évanescents. Le sujet remonte au début des années 70 : plus précisément, à des travaux de Langlands ([La]) et de Deligne ([De4]) visant à élucider le comportement aux mauvaises places des représentations galoisiennes associées aux formes modulaires. Ce problème, en ce qui concerne les représentations de dimension 2 associées aux formes classiques ou de Hilbert, a été résolu une dizaine d'années plus tard dans ma thèse ([Ca1], [Ca2]). Le cas de la dimension supérieure s'est alors posé et a donné lieu à divers travaux qui ont culminé dans la preuve par Harris et Taylor ([HT]) de la conjecture de Langlands locale pour $G L_{n}$. Ce résultat frappant n'a en rien clos le sujet; au contraire divers travaux plus récents et dont je parlerai brièvement ont encore enrichi la théorie et affiné de diverses façons les résultats de Harris et Taylor. En général, les espaces de cycles évanescents qui interviennent sont trop compliqués pour être explicitement calculés. L'idée fondamentale de Deligne est de relier cet espace "global" (lié aux formes automorphes et aux variétés de Shimura) à un espace "local" (lié à des représentations de groupes $p$-adiques et à des espaces de déformations de groupes formels), puis de jouer sur les 
relations entre le local et le global. A mon avis il s'agit de l'une des idées parmi les plus remarquables que Deligne a eues au cours de sa carrière scientifique (et aussi parmi celles qui ont eu une influence considérable), bien qu'elle soit sans doute moins connue que d'autres; lui même ne l'a exposée que dans une lettre manuscrite à Piatetskii-Shapiro, dans laquelle certaines questions techniques n'étaient pas résolues. D'ailleurs la méthode ne peut pleinement fonctionner (du moins dans le cas de variétés de dimension plus grande que 1) qu'avec la théorie des cycles évanescents de Berkovich. La théorie que nous allons exposer a atteint sa maturité pendant que Berkovich a développé la sienne relative aux espaces analytiques $p$-adiques et je pense que les besoins automorphes ont constitué pour ce dernier une motivation importante.

Je me limiterai dans cet exposé aux cas très particuliers où les espaces de cycles évanescents qui interviennent sont liés soit à cette construction de Deligne, soit à une autre, au bout du compte équivalente et due à Drinfeld. Le groupe local qui intervient est alors soit $G L_{n}$, soit le groupe multiplicatif d'un corps gauche d'invariant $1 / n$, avec $n$ entier. C'est actuellement essentiellement la seule situation où nous avons obtenu des résultats à peu près complets.

La théorie dans le cas de groupes plus généraux que $G L_{n}$ est l'objet d'un vaste programme initié par Rapoport et Zink il y a plus de dix ans. Mais ces groupes plus généraux posent des problèmes bien plus difficiles sur tous les plans. C'est pourquoi ce programme, bien que de nombreux résultats aient déjà été obtenus, est encore beaucoup moins avancé. Il en sera très peu question dans la suite.

\section{Le cas des courbes}

2.1. Représentations $\ell$-adiques associées aux formes modulaires. On sait, depuis le début des années 60 en poids 2 (Eichler - Shimura) et depuis la fin de cette même décennie pour les poids $\geq 2$ (Deligne), associer des représentations galoisiennes $\ell$-adiques aux formes modulaires. Plus précisément, soit $f=\sum a_{n} q^{n}$ une forme modulaire de poids $k \geq 2$, niveau $N$ et caractère "Nebentypus" $\epsilon$. Fixons d'autre part un plongement du corps $\mathbb{Q}(f)$, engendré par les coefficients de $f$, dans $\overline{\mathbb{Q}}_{\ell}$. Il existe alors une représentation continue:

$$
\sigma_{f, \ell}: \operatorname{Gal}(\overline{\mathbb{Q}} / \mathbb{Q}) \rightarrow G L_{2}\left(\overline{\mathbb{Q}}_{\ell}\right)
$$

qui est non-ramifiée en dehors de $N$ et telle que, pour $p$ ne divisant pas $N \ell$, on ait :

$$
(*) \operatorname{trace} \sigma_{f, \ell}\left(\operatorname{Frob}_{p}\right)=a_{p}, \operatorname{det} \sigma_{f, \ell}\left(\operatorname{Frob}_{p}\right)=p^{k-1} \epsilon(p) .
$$

Ces relations traduisent l'essentiel de ce que l'on peut dire du point de vue classique des relations entre $f$ et $\sigma_{f, \ell}$. Mais elles ne disent rien de ce qui se passe aux "mauvaises" places (lorsque $p$ divise $N$ ), ni ce qui arrive quand 
$p=\ell$. Dans cet exposé, je supposerai toujours $p \neq \ell$, car le cas où $p=\ell$ relève de techniques différentes.

De fait il était difficile du point de vue classique de formuler, même conjecturalement, ce qu'on attendait des propriétés de $\sigma=\sigma_{f, \ell}$ aux places $p \mid N$ : tout au plus conjecturait-on que le conducteur d'Artin de $\sigma$ devait être égal à $N$. A la fin des années soixante et au début de la décennie suivante les choses changent complètement avec le développement du programme de Langlands, qui permet de formuler une conjecture beaucoup plus précise.

A $f$ correspond une représentation automorphe $\pi=\otimes \pi_{v}$ de $G L_{2}(\mathbf{A})$ (où $\mathbf{A}$ désigne l'anneau des adèles de $\mathbb{Q}$ ) ; le produit tensoriel précédent est étendu à toutes les places de $\mathbb{Q}$ (nombres premiers et place archimédienne). Si $f$ est de poids $k$, la composante archimédienne $\pi_{\infty}$ est isomorphe à la représentation essentiellement de carré intégrable $\mathrm{DS}_{k}$ de $G L_{2}(\mathbb{R})$ qui apparaît dans la représentation de la série principale induite (induction unitaire) par les deux quasi-caractères suivants de $\mathbb{R}^{*}$ :

$$
\mu(t)=|t|^{k-\frac{1}{2}} \operatorname{sgn}(t) \quad, \quad \nu(t)=|t|^{\frac{1}{2}} .
$$

Pour chaque place finie $p, \pi_{p}$ est définie sur $\mathbb{Q}(f)$, et l'on a supposé choisi un plongement de ce corps dans $\overline{\mathbb{Q}}_{\ell}$.

Par ailleurs la correspondance de Langlands locale associe à chaque représentation (admissible, irréductible) de $G L_{2}\left(\mathbb{Q}_{p}\right)$ une représentation continue du groupe de Weil-Deligne local $W D_{\mathbb{Q}_{p}}$; ceci a un sens sur $\mathbb{C}$ mais aussi sur $\overline{\mathbb{Q}}_{\ell}$ et sur ce dernier corps on obtient plus simplement une représentation continue du groupe de Weil $W_{\mathbb{Q}_{p}}$, sous-groupe de $\operatorname{Gal}\left(\overline{\mathbb{Q}}_{p} / \mathbb{Q}_{p}\right)$ constitué des éléments qui induisent par réduction une puissance de Frobenius. Dans les années 70 cette conjecture n'était connue que dans certains cas; elle a été démontrée en toute généralité (avec $\mathbb{Q}_{p}$ remplacé par un corps local arbitraitre) par Kutzko en $1980([\mathbb{K u}])$.

Notons $\pi_{p} \rightarrow \sigma\left(\pi_{p}\right)$ la correspondance de Langlands locale, normalisée de telle sorte que les facteurs locaux $L$ et $\epsilon$ soient reliés par les identités (dans lesquelles $\chi$ désigne un quasi-caractère arbitraire de $\left.\mathbb{Q}_{p}^{*} \simeq W_{\mathbb{Q}_{p}}^{\mathrm{ab}}\right)$ :

$$
L\left(s, \sigma\left(\pi_{p}\right) \otimes \chi\right)=L\left(s-\frac{1}{2}, \pi_{p} \otimes \chi\right), \quad \epsilon\left(s, \sigma\left(\pi_{p}\right) \otimes \chi\right)=\epsilon\left(s-\frac{1}{2}, \pi_{p} \otimes \chi\right) ;
$$

elle diffère par une torsion de la correspondance usuelle.

Le résultat suivant a été conjecturé dans les années 70 et démontré en 1984 :

Théorème 2.1. - ([La], [De4], [Ca2]) Pour tout $p \neq \ell$, la (Frobenius semisimplifiée de la) restriction de $\sigma_{f, \ell}$ au groupe de decomposition en $p$, ou plus précisément au groupe de Weil $W_{\mathbb{Q}_{p}}$, est isomorphe à $\sigma\left(\pi_{p}\right)$. 
Le cas non ramifié correspond au cas où $\pi_{p}$ est une série principale non ramifiée (induite de deux caractères non ramifiés de $\mathbb{Q}_{p}^{*}$ ) et il n'est pas difficile alors de voir que l'énoncé dans ce cas est équivalent aux égalités $\left(^{*}\right)$ ci-dessus. Une avancée esssentielle est due à Langlands dans son article [La] : il a prouvé le résultat quand $\pi_{p}$ est une série principale ramifiée ou bien une représentation spéciale. Peu de temps après, Deligne (dans une lettre à Piatetskii-Shapiro) a compris le cas des représentations cuspidales ordinaires (induites par induction automorphe à partir d'un caractère d'une extension quadratique - ce qui s'appelait à l'époque construction de Weil). Nous allons maintenant donner une idée des méthodes employées par Langlands et Deligne et expliquer comment intervient la théorie des cycles évanescents.

\subsection{Géométrie des courbes modulaires et méthode de Langlands.}

- La construction des représentations $\ell$-adiques dont il vient d'être question se fait en décomposant la cohomologie $\ell$-adique des courbes modulaires sous l'action des opérateurs de Hecke.

Classiquement, les courbes modulaires $X_{0}(N), X_{1}(N), X(N)$ sont les quotients du demi-plan de Poincaré par certains sous-groupe de congruence de $S L_{2}(\mathbb{Z})$. Du point de vue de la théorie automorphe moderne (adélique) on préfère partir d'un sous-groupe compact et ouvert $K$ du groupe $G L_{2}\left(\mathbf{A}_{f}\right)$ des points de $G L_{2}$ à valeurs dans les adèles finies, et considérer le quotient :

$$
M_{K}(\mathbb{C})=G L_{2}(\mathbb{Q}) \backslash X \times G L_{2}\left(\mathbf{A}_{f}\right) / K,
$$

avec $X=\mathbb{C}-\mathbb{R}$ (double demi-plan de Poincaré). Ce quotient n'est rien d'autre (pour $K$ assez petit) qu'une réunion finie de quotients du demi-plan de Poincaré par des sous-groupes de congruence de $S L_{2}(\mathbb{Z})$.

Ces objets, à priori des surfaces de Riemann, admettent des structures de variétés algébriques sur $\mathbb{C}$ et sont en fait naturellement des courbes $M_{K}$ définies sur $\mathbb{Q}$, qui possèdent aussi des compactifications (lisses) $\bar{M}_{K}$. Elles constituent de façon naturelle un système projectif lorsque $K$ varie : cela est clair du point de vue analytique et il se trouve que les morphismes du système sont définis sur $\mathbb{Q}$.

La construction des représentations galoisiennes attachées aux formes automorphes peut alors s'exprimer par l'égalité suivante qui exprime la décomposition de la (limite inductive de la) cohomologie $\ell$-adique sous la double action du groupe de Galois et du groupe $G L_{2}\left(\mathbf{A}_{f}\right)$ :

$$
(* *) \underset{K}{\lim _{\longrightarrow}} H^{1}\left(\bar{M}_{K} \otimes \mathbb{Q} \overline{\mathbb{Q}}, \overline{\mathbb{Q}}_{\ell}\right)=\bigoplus_{\pi} \pi_{f} \otimes \sigma(\pi),
$$

où la somme est étendue aux représentations automorphes dont la composante archimédienne est $D S_{2}$ (autrement dit il s'agit du cas des formes de poids 2). On obtient une construction analogue pour les formes de poids $k$ en remplaçant 
ci-dessus le faisceau constant $\overline{\mathbb{Q}}_{\ell}$ par un système local $\ell$-adique convenable et en considérant les groupes de cohomologie d'intersection (plus simplement ici, si l'on préfère, l'image de la cohomologie à support dans la cohomologie).

Dans la suite et pour simplifier, je ne considérerai que le faisceau constant et donc les formes de poids 2 . Le cas général ne présente que de faibles différences techniques, peu importantes du point de vue de cet exposé.

Notons $M_{n, H}$ la courbe $M_{K}$ lorsque $K$ est le produit du sous-groupe de congruence principal de niveau $n$ dans $G L_{2}\left(\mathbb{Z}_{p}\right)$ (constitué des matrices congrues à 1 modulo $p^{n}$ ) par un sous-groupe $H$ de $G L_{2}\left(\mathbf{A}_{f}^{p}\right)$. Notation analogue $\bar{M}_{n, H}$ pour la compactifiée. Lorsque $n=0$ cette courbe a bonne réduction en $p$ : sa cohomologie coïncide donc avec celle de sa fibre en $p$, dont l'ensemble des points (muni de l'action de Frobenius et des opérateurs de Hecke) admet une description explicite. Cela permet d'obtenir le cas classique : déterminer la trace de Frobenius en un $p$ qui ne divise pas le niveau de la forme modulaire. En termes adéliques comme dans la formule ci-dessus, on récupère ainsi les représentations automorphes $\pi$ telles que $\pi_{p}$ soit une représentation de la série principale sphérique (induite de deux quasi-caractères non-ramifiés).

Dans le cas général on montre que $M_{n, H}$ peut s'obtenir par restriction des scalaires à partir d'une courbe définie sur l'extension cyclotomique $\mathbb{Q}_{p^{n}}$ engendrée par les racines $p^{n}$-ièmes de l'unité. On dispose d'un modèle $\mathbf{M}_{n, H}$ de $M_{n, H}$ sur l'anneau des entiers $\mathbb{Z}_{p^{n}}$ de $\mathbb{Q}_{p^{n}}$ et ce modèle est lisse sauf aux points supersinguliers (le schéma $\mathbf{M}_{n, H}$ est un espace de modules pour des courbes elliptiques munies de certaines structures et les points supersinguliers sont les points de la fibre spéciale correspondant à des courbes elliptiques supersingulières). De plus la fibre spéciale de $\mathbf{M}_{n, H}$ est réduite et c'est une réunion de composantes irréductibles lisses et qui s'intersectent aux points supersinguliers seulement. On a un compactifié $\overline{\mathbf{M}}_{n, H}$ qui vérifie des propriétés semblables.

On s'intéresse aux groupes de cohomologie $\mathcal{H}^{n, H}$ de :

$$
\bar{M}_{n, H} \otimes \overline{\mathbb{Q}}_{p}=\bigsqcup_{\iota} \bar{M}_{n, H} \otimes_{\mathbb{Q}_{p}, \iota} \overline{\mathbb{Q}}_{p}
$$

$\iota$ décrivant l'ensemble des plongements de $\mathbb{Q}_{p^{n}}$ dans $\overline{\mathbb{Q}}_{p}$. Si l'on considère la réunion :

$$
\overline{\mathbf{M}}_{n, H} \bar{\otimes} \overline{\mathbb{Z}}_{p}={ }_{\text {def }} \bigsqcup_{\iota} \overline{\mathbf{M}}_{n, H} \otimes_{\mathbb{Z}_{p^{n}, \iota}} \overline{\mathbb{Z}}_{p}
$$

et si nous notons $\overline{\mathbf{M}}_{n, H} \bar{\otimes} \overline{\mathbb{F}}_{p}$ sa fibre spéciale, alors la théorie des cycles évanescents produit la suite exacte suivante:

$$
0 \rightarrow \mathcal{H}_{1}^{n, H} \rightarrow \mathcal{H}^{n, H} \rightarrow \mathcal{H}_{2}^{n, H}
$$

avec $\mathcal{H}_{1}^{n, H}=H^{1}\left(\overline{\mathbf{M}}_{n, H} \bar{\otimes} \overline{\mathbb{F}}_{p}, \overline{\mathbb{Q}}_{\ell}\right)$ et où $\mathcal{H}_{2}^{n, H}$ est la somme, pour tous les points supersinguliers $x$ de la fibre spéciale, des groupes de cycles évanescents 
$R \Phi_{x}^{1}$ : pour un tel $x$, l'espace $R \Phi_{x}^{1}$ est par définition la cohomologie de la fibre générique géométrique $\left[X_{(x)}\right] \bar{\eta}$ de l'henselisé strict de $X$ en $x$.

La flèche de droite est "presque" surjective (le groupe $G L_{2}\left(\mathbf{A}_{f}\right)$ opère sur son conoyau via le déterminant, de sorte qu'aucune forme parabolique n'y intervient).

Notons $\mathcal{H}_{1}$ (resp. $\mathcal{H}_{2}$, resp. $\mathcal{H}$ ) les limites inductives, quand $n$ et $H$ varient, de ces espaces de cohomologie.

Langlands a utilisé dans [La] une comparaison entre les formules de traces de Selberg et de Lefschetz (compte tenu du fait qu'il est possible d'avoir une description combinatoire de l'ensemble des points points de $\mathbf{M}_{n, H} \bar{\otimes} \overline{\mathbb{F}}_{p}$ ) et il a ainsi calculé $\mathcal{H}_{1}$. Il est également possible de retrouver le même résultat à partir d'une version de la relation de congruence, due à Piatetskii-Shapiro ([PS]). Langlands a ainsi obtenu la description suivante de cet espace, muni des actions de $G\left(\mathbf{A}_{f}\right)$ et $\operatorname{Gal}\left(\overline{\mathbb{Q}}_{p} / \mathbb{Q}_{p}\right)$ :

$$
\mathcal{H}_{1}=\bigoplus_{\pi} \pi_{f} \otimes \sigma^{\prime}\left(\pi_{p}\right)
$$

où $\pi$ décrit l'ensemble des représentations automorphes cuspidales de $G L_{2}(\mathbf{A})$ telles que $\pi_{\infty} \simeq \mathrm{DS}_{2}$ et que $\pi_{p}$ soit une représentation de la série principale (incluant les ramifiées) ou bien une représentation spéciale. Dans cette formule on a $\sigma^{\prime}\left(\pi_{p}\right)=\sigma\left(\pi_{p}\right)$ quand $\pi_{p}$ est principale, tandis que, lorsque $\pi_{p}$ est spéciale, $\sigma^{\prime}\left(\pi_{p}\right)$ est seulement la "moitié" de la représentation spéciale $\sigma\left(\pi_{p}\right)$, i.e. son sous-espace invariant de dimension 1.

L'article de Langlands réglait ainsi le cas où la composante locale $\pi_{p}$ était une représentation de la série principale (ramifiée ou non) ou bien spéciale; le cas des cuspidales, qui se retrouvent donc toutes, d'après ce qui précède, dans la partie évanescente $\mathcal{H}_{2}$ de la cohomologie, restait complètement mystérieuse : aucun calcul direct ne semble possible car pour $n$ grand les courbes modulaires présentent des singularités très compliquées.

2.3. L'idée de Deligne. - L'idée qu'a eue Deligne, peu de temps après, fut de contourner cette difficulté majeure en reliant la cohomologie évanescente à une représentation $\mathcal{U}$ ("représentation locale fondamentale") du produit triple $G L_{2}\left(\mathbb{Q}_{p}\right) \times D_{p}^{*} \times W_{\mathbb{Q}_{p}}$ où $D_{p}$ désigne le corps de quaternions (unique à isomorphisme près) de centre $\mathbb{Q}_{p}$ et $W_{\mathbb{Q}_{p}}$ le groupe de Weil. Cette représentation est construite de façon purement locale en considérant des déformations de groupes formels.

Plus précisément, soit $\Sigma$ un groupe formel de dimension 1 et de hauteur 2 sur $\overline{\mathbb{F}}_{p}$ (un tel groupe est unique à isomorphisme près). L'anneau $\operatorname{End}(\Sigma) \otimes \mathbb{Q}$ est isomorphe à $D_{p}$.

Les déformations de $\Sigma$ munies d'une structure de niveau $p^{n}$ (ce qui signifie, au niveau du moins de la fibre générique, une trivialisation de la $p^{n}$-torsion 
du groupe) sont représentées par un schéma $\operatorname{Def}_{\Sigma}^{n}$, qui admet une structure

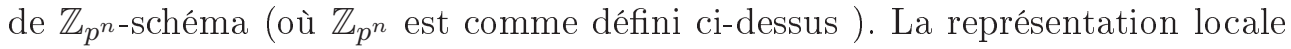
fondamentale est construite à partir de la cohomologie de la fibre générique géométrique de ce schéma :

$$
\Phi_{\Sigma}^{n}=H^{1}\left(\operatorname{Def}_{\Sigma}^{n} \otimes_{\mathbb{Z}_{p^{n}}} \overline{\mathbb{Q}}_{p}, \overline{\mathbb{Q}}_{\ell}\right)
$$

Cependant, afin de tenir compte des actions des différents groupes en jeu, il est préférable de partir d'un groupe formel qui est seulement défini " à isogénie près". Il est alors possible de parler de déformations et de structures de niveau pour un tel objet. Je ne vais pas expliquer ici les détails précis de ces constructions, pour lesquels je renverrai à [Ca2]. Je vais simplement exprimer, de façon un peu vague, ce que l'on obtient, puis j'expliquerai comment cela est utilisé pour résoudre le problème posé.

Ces constructions fournissent finalement un espace de paramètres $\Delta \simeq \mathbb{Q}_{p}^{*}$ et pour chaque $x \in \Delta$ un espace vectoriel $\Phi_{x}^{n}$ comme ci-dessus (ces espaces constituent une sorte de faisceau sur $\Delta$ ). Sur $\Delta$ agissent les trois groupes $G L_{2}\left(\mathbb{Q}_{p}\right)$ (via det), $D_{p}^{*}$ (via l'inverse de la norme réduite), et finalement $W_{\mathbb{Q}_{p}}$ (via l'inverse de l'homomorphisme de la théorie du corps de classes); ces trois actions se relèvent au système des $\Phi_{x}$, limite inductive des $\Phi_{x}^{n}$.

La représentation locale fondamentale est l'espace des sections :

$$
\mathcal{U} \subset H^{0}(\Delta, \Phi)
$$

où $\Phi$ désigne le "faisceau" sur $\Delta$ constitué des $\Phi_{x}$ définis ci-dessus, et qui sont invariantes sous un sous-groupe ouvert du centre de $G L_{2}\left(\mathbb{Q}_{p}\right)$ (ceci afin d'avoir une représentation "raisonnable").

Il est classique de décrire l'ensemble des points supersinguliers de la fibre spéciale $\varliminf_{n} \mathbf{M}_{n, H} \bar{\otimes} \overline{\mathbb{F}}_{p}$ comme le quotient

$$
M^{\mathrm{ss}}=\bar{G}(\mathbb{Q}) \backslash \Delta \times \bar{G}\left(\mathbf{A}_{f}^{p}\right) / H
$$

où l'on a noté $\bar{G}$ le groupe multiplicatif $D^{*}$ de l'algèbre de quaternions $D$ sur $\mathbb{Q}$ qui n'est ramifiée qu'en $p$ et $\infty$ - on remarquera que $\bar{G}\left(\mathbf{A}_{f}^{p}\right)$ est isomorphe à $G\left(\mathbf{A}_{f}^{p}\right)$; on fixera un tel isomorphisme, ce qui nous permettra dans la suite d'identifier ces deux groupes.

On démontre alors qu'une description similaire (sous forme d'un quotient) est aussi valide pour le "faisceau" (au-dessus de l'ensemble des points supersinguliers) $R \Phi^{1}$ des cycles évanescents : on a une identification

$$
R \Phi^{1}=\bar{G}(\mathbb{Q}) \backslash \Phi \times \bar{G}\left(\mathbf{A}_{f}^{p}\right) / H
$$

On obtient cela en utilisant le théorème de Serre et Tate (qui affirme que les déformations formelles d'une courbe elliptique correspondent à celles du groupe 
p-divisible sous-jacent), mais on a besoin en plus d'un résultat permettant de comparer les cycles évanescents ordinaires et formels. Pour les courbes un tel résultat a été prouvé par Brylinski (dans un appendice à ma thèse -voir cidessous); des résultats analogues (quoique légèrement différents) en dimension plus grande ont été obtenus par Berkovich et j'en parlerai dans la deuxième partie de cet article.

On peut donc voir (à quelques détails techniques près que je négligerai dans la suite) $\mathcal{H}_{2}$ comme l'ensemble des applications :

$$
f: \bar{G}\left(\mathbf{A}_{f}^{p}\right) \rightarrow \mathcal{U}
$$

qui sont $\bar{G}(\mathbb{Q})$-équivariantes à gauche et invariantes à droite sous un sousgroupe compact ouvert de $\bar{G}\left(\mathbf{A}_{f}^{p}\right)$. A une telle $f$ on associe alors l'application suivante $f^{\prime}$ de $\bar{G}(\mathbf{A})$ vers $\mathcal{U}$ :

$$
f^{\prime}\left(g_{f}^{p} g_{p} g_{\infty}\right)=g_{p}^{-1} \cdot f\left(g_{f}^{p}\right)
$$

laquelle est maintenant invariante à gauche sous $\bar{G}(\mathbb{Q})$ et à droite invariante sous $\bar{G}(\mathbb{R})$ et équivariante sous l'action de $\bar{G}\left(\mathbb{Q}_{p}\right)$. On obtient de cette manière un isomorphisme :

$$
\mathcal{H}_{2} \simeq\left(\overline{\mathcal{A}}_{1} \otimes \mathcal{U}\right)^{\bar{G}\left(\mathbb{Q}_{p}\right)}
$$

où $\overline{\mathcal{A}}_{1}$ désigne l'espace des formes automorphes sur $\bar{G}$ de composante archimédienne triviale. Ce dernier espace se décompose comme une somme $\bigoplus \bar{\pi}$ de représentations automorphes irréductibles et nous obtenons la formule :

$$
\mathcal{H}_{2}=\bigoplus \bar{\pi}_{f}^{p} \otimes \mathcal{U}\left(\bar{\pi}_{p}^{\vee}\right)
$$

où la somme est étendue à l'ensemble des $\pi$ automorphes de composante archimédienne triviale et où $\mathcal{U}\left(\bar{\pi}_{p}^{\vee}\right)$ désigne la composante isotypique (relative à l'action de $\left.\bar{G}\left(\mathbb{Q}_{p}\right)\right)$ correspondant à la contragrédiente de $\bar{\pi}_{p}$.

On utilise alors la correspondance de Jacquet-Langlands entre représentations de $G$ et celles de $\bar{G}$. Localement en un nombre premier $q$, c'est une bijection entre représentations de $\bar{G}\left(\mathbb{Q}_{q}\right)$ et représentations de la série discrète de $G\left(\mathbb{Q}_{q}\right)$ et elle se globalise en une correspondance entre représentations automorphes de $\bar{G}(\mathbf{A})$ et de $G(\mathbf{A})$ : à $\bar{\pi}=\otimes_{q \leq \infty} \bar{\pi}_{q}$ on associe $\pi=\otimes_{q \leq \infty} \pi_{q}$ avec $\pi_{q}=\bar{\pi}_{q}$ aux places $q$ où $D$ n'est pas ramifiée (donc ici toutes les places sauf $p$ et $\infty$ ) tandis que sinon c'est la représentation de $G\left(\mathbb{Q}_{q}\right)$ qui correpond à $\bar{\pi}_{q}$ par la correspondance locale. On montre alors que $\pi$ est automorphe lorsque $\bar{\pi}$ l'est, et qu'on obtient ainsi toutes les représentations automorphes de $G(\mathbf{A})$ dont les composantes locales aux places ramifiées appartiennent à la série discrète. 
Utilisant cette correspondance ainsi que la formule $(* *)$ ci-dessus, on conclut de ce qui précède que, pour $\pi_{p}$ cuspidale, la représentation galoisienne correspondante restreinte au groupe de Weil local en $p$ est donnée par :

$$
\pi_{p} \otimes \sigma(\pi)_{\mid \operatorname{Gal}\left(\overline{\mathbb{Q}}_{p} / \mathbb{Q}_{p}\right)}=\mathcal{U}\left(\bar{\pi}_{p}^{\vee}\right)
$$

où $\bar{\pi}_{p}$ désigne la représentation de $\bar{G}\left(\mathbb{Q}_{p}\right)$ qui correspond à $\pi_{p}$ par la correspondance locale de Jacquet-Langlands.

Le point crucial est que $\sigma(\pi)_{\mid \operatorname{Gal}\left(\overline{\mathbb{Q}}_{p} / \mathbb{Q}_{p}\right)}$ ne dépend que de $\pi_{p}$. Une fois qu'on sait cela il est facile de traiter le cas des représentations dites "ordinaires" : il s'agit de celles obtenues à partir d'un quasi-caractère du groupe multiplicatif d'une extension quadratique de $\mathbb{Q}_{p}$ par une construction due à Weil (et maintenant généralisée par ce que l'on appelle "l'induction automorphe"). Cette construction locale admet un avatar global. Tant du point de vue local que global, ces constructions constituent le pendant automorphe de l'induction à partir du groupe de Galois d'une extension quadratique. Si maintenant $\pi_{p}$ est une représentation de Weil locale, alors on est ramené au cas où $\pi$ est globalement obtenue à partir d'un caractère de Hecke d'un corps quadratique. Il n'est pas difficile alors de voir (puisque la situation est controlée en presque chaque place) que la représentation galoisienne est globalement induite; par restriction au groupe de Galois local en $p$ on obtient alors ce qu'on veut.

2.4. Courbes de Shimura. - À la suite de cette lettre informelle de Deligne, il restait deux questions non réglées : d'une part le problème technique de comparer cycles évanescents usuels et formels (i.e. définis à partir du complété formel) ; d'autre part la question des représentations "extraordinaires" (non induites) était restée ouverte. Ces deux questions ont été résolues dans ma thèse (1984). Dans un appendice à ce travail, Brylinski avait utilisé un argument utilisant la résolution des singularités (pour les courbes) pour prouver l'égalité entre cycles évanescents usuels et formels, plus quelques résultats de continuité nécessaires au fonctionnement correct de l'argument de Deligne.

Ma contribution personnelle a consisté à étudier les courbes de Shimura pour découvrir que leurs réductions pouvaient être décrites par une théorie très semblable à celle des courbes modulaires. Une telle courbe est définie à partir d'un corps totalement réel $F$, muni d'un plongement $\tau: F \hookrightarrow \mathbb{R}$, et d'une algèbre de quaternions $B / F$ qui est déployée à la place $\tau$ et ramifiée aux autres places archimédiennes. On désigne par $G$ le groupe multiplicatif de $B$, vu comme groupe algébrique sur $\mathbb{Q}: \quad G=\operatorname{Res}_{F / Q}\left(B^{*}\right)$. Le groupe $G(\mathbb{R})$ est isomorphe au produit $G L_{2}(\mathbb{R}) \times\left(\mathbf{H}^{*}\right)^{d-1}$, où $\mathbf{H}$ designe le corps des quaternions de Hamilton et il opère via son premier facteur sur $X=\mathbb{C}-\mathbb{R}$ (comme plus haut, le "double" demi-plan de Poincaré ). 
Pour $K$ un sous-groupe compact et ouvert du groupe $G\left(\mathbf{A}_{f}\right)$, la courbe de Shimura associée peut être définie analytiquement comme le quotient analogue à celui qui définissait les courbes modulaires :

$$
M_{K}(\mathbb{C})=G(\mathbb{Q}) \backslash X \times G\left(\mathbf{A}_{f}\right) / K,
$$

et ce n'est d'ailleurs rien d'autre qu'une réunion finie disjointe de quotients $\Gamma_{i} \backslash X^{+}$du demi-plan de Poincaré par des sous-groupes discrets de $S L_{2}(\mathbb{R})$. On retrouve les courbes modulaires lorsque $F=\mathbb{Q}$ et que $B$ est déployée, tandis que dans les autres cas les courbes obtenues sont propres.

D'après Shimura, ces courbes admettent un modèle "canonique" sur le corps $F$ (plongé dans $\mathbb{C}$ via $\tau$ ). Pour $K$ variant, elles constituent un système projectif de courbes sur $F$.

Comme dans le cas des courbes modulaires, on montre que l'on peut utiliser ces courbes pour construire des représentations $\ell$-adiques de dimension $2 \mathrm{du}$ groupe $\operatorname{Gal}(\bar{F} / F)$ : on décompose pour cela la limite inductive des groupes de cohomologie $\ell$-adique et l'on obtient la formule suivante analogue à la formule $(* *)$ ci-dessus :

$$
(* * *) \underset{K}{\lim _{\longrightarrow}} H^{1}\left(M_{K} \otimes_{F} \bar{F}, \overline{\mathbb{Q}}_{\ell}\right)=\bigoplus_{\pi} \pi_{f} \otimes \sigma(\pi)
$$

la somme étant étendue aux représentations automorphes de $G(\mathbf{A})$ de composante archimédienne triviale aux places $\neq \tau$ et telles que $\pi_{\tau} \simeq \mathrm{DS}_{2}$.

Cela donne une construction de représentations galoisiennes $\sigma(\pi)$ de degré 2 associées aux $\pi$ qui interviennent. Via la correspondance de Jacquet-Langlands, ces dernières correspondent à des formes modulaires de Hilbert de poids parallèle 2. En remplaçant le faisceau constant par des systèmes locaux convenables, on peut obtenir des composantes archimédiennes (des poids) plus générales.

Une des dificultés techniques essentielles que l'on rencontre lorsque l'on tente d'étudier la réduction de ces courbes de Shimura est qu'elles ne représentent pas de façon naturelle un problème de modules (défini en termes de schémas abéliens polarisés munis de certaines actions et de certaines structures de niveau). Ce n'est toutefois pas loin d'être le cas : il existe une famille de groupes $G^{\prime}$ (admettant le même groupe dérivé que $G$ ) telles que les courbes de Shimura $M_{K^{\prime}}^{\prime}$ définies à partir de $G^{\prime}$ plutôt que de $G$ soient solution d'un tel problème de modules, mais défini en général sur un corps $F^{\prime}$ différent de $F$. Les courbes $M_{K^{\prime}}^{\prime}$ sont très proches des $M_{K}$ : elles ont, en un sens que je ne vais pas chercher à préciser, les mêmes composantes connexes. On peut reconstituer assez facilement (bien que cela soit techniquement embrouillé) $M_{K}$ par une sorte de recollement des $M_{K^{\prime}}^{\prime}$.

$\mathrm{Au}$ bout du compte, on s'aperçoit en étudiant ainsi la réduction d'un modèle entier des $M_{K} \otimes F_{\mathfrak{p}}$ en une place $\mathfrak{p}$ de $F$ où $B$ est déployée qu'il existe sur ce modèle (ou plutôt sur une limite projective convenable) un groupe $p$-divisible 
$\mathbf{E}$ défini de façon canonique et qui joue le rôle du groupe $p$-divisible associé à la courbe elliptique universelle sur les courbes modulaires. Ce groupe est en fait muni d'une action de l'anneau $\mathcal{O}_{\mathfrak{p}}$ des entiers de $F_{\mathfrak{p}}$ et il constitue ce que Drinfeld avait nommé un " $\mathcal{O}_{\mathfrak{p}}$-module divisible". En un point $x$ de la fibre spéciale deux cas sont possibles : ou bien $\mathbf{E}_{x}$ est isomorphe à une extension d'un groupe de Lubin-Tate par le module constant $F_{\mathfrak{p}} / \mathcal{O}_{\mathfrak{p}}$ (cas ordinaire), ou bien ce groupe est isomorphe à l'unique module infinitésimal de hauteur 2 (voir plus bas pour des définitions plus précises). Tout cela généralise des choses bien connues dans le cas des courbes modulaires. Les points supersinguliers de ces courbes constituent des ensembles finis descriptibles de façon analogue à ce qu'on a dit plus haut dans le cas des courbes modulaires. De plus on a un avatar du théorème de Serre et Tate qui permet de calculer le complété formel en un point de nos courbes comme un espace de déformations (avec structures de niveau) du $\mathcal{O}_{\mathfrak{p}}$-module divisible correspondant. Ces similarités permettent d'adapter les méthodes de Langlands et de Deligne et de prouver les résultats voulus en une place $\mathfrak{p}$ où $\pi_{\mathfrak{p}}$ est soit principale, soit cuspidale ordinaire.

2.5. Représentations extraordinaires. - Expliquons maintenant comment prouver le théorème ci-dessus à partir de ce qui précède. Le cas qu'il nous reste à traiter est celui où $\pi_{p}$ est une représentation cuspidale extraordinaire et cela correspond au fait que $\sigma\left(\pi_{p}\right)$ est "primitive" (non induite par un caractère du groupe de Galois d'une extension quadratique de $\mathbb{Q}_{p}$ ). Les représentations induites ont pour image dans le groupe $P G L_{2}$ un sous-groupe diédral. Les représentations primitives de degré 2 ont une image projective isomorphe soit au groupe alterné $\mathcal{A}_{4}$ soit au groupe symétrique $\mathcal{S}_{4}$ (le cas $\mathcal{A}_{5}$ étant exclu puisque les groupes de Galois locaux sont résolubles).

Chacun des deux groupes possibles admet des 2-sous-groupes de Sylow qui sont des groupes diédraux; plus précisément il en existe un seul (distingué) dans le cas $\mathcal{A}_{4}$ tandis qu'on en a 3 dans le cas $\mathcal{S}_{4}$ et l'on choisit arbitrairement l'un des 3 .

Dans les deux cas il correspond à ce sous-groupe de Sylow, qui est d'indice 3, une extension cubique $F_{\mathfrak{p}}$ de $\mathbb{Q}_{p}$, qui est galoisienne dans le cas $\mathcal{A}_{4}$ mais non dans le cas $\mathcal{S}_{4}$. La théorie automorphe produit un foncteur de changement de base qui à une représentation (admissible, irréductible) de $G L_{2}\left(\mathbb{Q}_{p}\right)$ en associe une de $G L_{2}\left(F_{\mathfrak{p}}\right)$. Localement ce n'est rien d'autre que ce qui correspond, via la correpondance de Langlands locale, à la restriction d'une représentation galoisienne de $W D_{\mathbb{Q}_{p}}$ à $W D_{F_{\mathfrak{p}}}$, et ces foncteurs aux différentes places se globalisent en une opération qui fait passer d'une représentation automorphe de $G L_{2}\left(\mathbf{A}_{\mathbb{Q}}\right)$ à une autre de $G L_{2}\left(\mathbf{A}_{F}\right)$, pour $F$ une extension cubique de $\mathbb{Q}$ (habituellement on ne sait faire ce changement de base que dans le cas des extensions galoisiennes cycliques, le cas cubique non galoisien constituant une exception et résultant de travaux de Jacquet, Piatetskii-Shapiro et Shalika). 
On choisit alors un corps cubique totalement réel $F$ tel que $F \otimes \mathbb{Q}_{p} \simeq F_{\mathfrak{p}}$. Notons $\Pi$ la représentation automorphe de $G L_{2}\left(\mathbf{A}_{F}\right)$ associée à $\pi$ par changement de base. Alors $\Pi_{\mathfrak{p}}$, associée à la restriction (diédrale d'après le choix de $F_{\mathfrak{p}}$ ) de $\sigma\left(\pi_{p}\right)$ au groupe de Weil de $F_{\mathfrak{p}}$, est une représentation ordinaire. De ce qui précède on déduit que la représentation galoisienne $\ell$-adique associée à $\pi$, qui n'est autre bien sûr que la restriction de $\sigma_{f, \ell}$ au groupe de Galois de $F$, est ce que l'on espère à la place $\mathfrak{p}$. On conclut alors en remarquant (c'est un simple exercice de théorie des groupes) que la représentation $\sigma\left(\pi_{\mathfrak{p}}\right)$ est caractérisée par sa restriction à $\operatorname{Gal}\left(\bar{F}_{\mathfrak{p}} / F_{\mathfrak{p}}\right)$ et par son déterminant (qui est connu ici).

Pour conclure ce paragraphe, mentionnons que l'on a un analogue du théorème précédent pour les représentations associées aux formes modulaires de Hilbert sur des corps totalement réels et que ce théorème se démontre de façon analogue. Dans le cas du corps $\mathbb{Q}$ on a une preuve différente, due à Louise Nyssen $[\mathrm{Ny}]$, qui ne fait pas usage des courbes de Shimura. Elle utilise plutôt un argument de congruence avec des formes de poids 1 .

\section{Le cas de la dimension supérieure}

\subsection{La représentation locale fondamentale en dimension supérieure.}

- La généralisation de notre représentation locale en dimension supérieure n'est pas très difficile dans son principe : il suffit de considérer des modules formels de hauteur supérieure à 2 .

Donnons quelques définitions plus précises que ce que nous avons fait jusqu'alors. Soit $K$ un corps local non archimédien, de corps résiduel $k$ fini dont on note $p$ la caractéristique et $q$ le cardinal. K est donc soit un corps $p$-adique (extension finie de $\mathbb{Q}_{p}$ ) soit isomorphe au corps $\mathbb{F}_{q}((t))$ des séries de Laurent formelles en une variable. Notons $\mathcal{O}$ l'anneau des entiers de $K$ et fixons une uniformisante $\varpi$. Nous désignerons par $\hat{\mathcal{O}}^{\mathrm{nr}}$ l'anneau des entiers du complété d'une extension non-ramifiée maximale $K^{\text {nr }}$ de $K$.

On appelle $\mathcal{O}$-module formel (de dimension 1) sur une $\mathcal{O}$-algèbre $R$ un groupe formel $\Sigma$ de dimension 1, muni d'une action de l'anneau $\mathcal{O}$, de telle sorte que l'action dérivée sur Lie $(\Sigma)$ soit donnée par le morphisme structural $\mathcal{O} \rightarrow R$. Dans le cas où $R$ est un corps extension du corps résiduel $k$ de $K$, on peut définir la hauteur $h$ de $\Sigma$ de la façon suivante : une coordonnée $X$ sur le groupe formel étant choisie, $q^{h}$ est alors l'ordre de la série formelle en $X$ qui exprime l'action de $\varpi$ (dans la suite cet ordre et donc la hauteur seront toujours finis). On ne confondra pas cette notion avec la hauteur de $\Sigma$ vu simplement comme groupe formel (dans le cas où $K$ est un corps $p$-adique, la hauteur du groupe est égale à $\left.h . \operatorname{deg}\left[K: \mathbb{Q}_{p}\right]\right)$. Lorsque $K=\mathbb{Q}_{p}$, un $\mathbb{Z}_{p}$-module formel n'est rien de plus qu'un groupe formel. Ces objets ont été étudiés par Drinfeld ([Dr1]) au début des années 70 dans son travail sur les "modules elliptiques". Les résultats habituels 
sur les groupes formels se transposent sans difficulté. En particulier, sur $\bar{k}$, clôture algébrique du corps résiduel de $K$, il existe un unique (à isomorphisme près) module formel $\Sigma_{h}$ de hauteur $h$. L'anneau des endomorphismes de $\Sigma_{h}$ est isomorphe à l'ordre maximal du corps gauche $D_{K, h}$ de centre $K$, de dimension $h^{2}$ et d'invariant $1 / h$ dans le groupe de Brauer.

On s'intéresse à la théorie des déformations de $\Sigma_{h}$, autrement dit au foncteur Def qui à une $\hat{\mathcal{O}}^{\text {nr }}$-algèbre artinienne $R$ de corps résiduel $\bar{k}$ associe l'ensemble des classes d'isomorphisme de déformations de $\Sigma_{h}$ sur $R(\mathcal{O}$-modules formels sur $\mathrm{R}$ munis d'un isomorphisme entre leur réductions modulo l'idéal maximal de $R$ et $\Sigma_{h}$ ). On peut montrer que Def est (pro-)représentable par un schéma formel que nous noterons $\operatorname{Def}_{\Sigma}^{0}$; on a en fait un isomorphisme

$$
\operatorname{Def}_{\Sigma}^{0} \simeq \operatorname{Spf}\left(\hat{\mathcal{O}}^{\mathrm{nr}}\left[\left[T_{1}, T_{2}, \ldots T_{h-1}\right]\right]\right)
$$

Nous noterons $\tilde{\Sigma}_{h}$ la déformation universelle de $\Sigma_{h}$.

On définit pour chaque entier $m \geq 1$ un schéma formel $\operatorname{Def}_{\Sigma}^{m}$ au-dessus de $\operatorname{Def}_{\Sigma}^{0}$ comme le classifiant des structures de niveau $m$ sur le groupe $\tilde{\Sigma}_{h}$. Au-dessus de la fibre générique de $\operatorname{Def}_{\Sigma}^{0}$, une telle structure est simplement la donnée d'un isomorphisme entre $\left(\varpi^{-m} \mathcal{O} / \mathcal{O}\right)^{h}$ et la $\varpi^{m}$-torsion du module formel, et on a la notion de "base de Drinfeld" (qu'il est peu important de connaître précisément pour la suite de cet exposé) qui permet de définir cette notion même sur la fibre spéciale.

Lorsque $m^{\prime}>m$ on a une inclusion évidente de $\left(\varpi^{-m} \mathcal{O} / \mathcal{O}\right)^{h}$ dans $\left(\varpi^{-m^{\prime}} \mathcal{O} / \mathcal{O}\right)^{h}$ qui permet de "restreindre" une base de niveau $m^{\prime}$ en une de niveau $m$. Cela définit une projection de $\operatorname{Def}_{\Sigma}^{m^{\prime}}$ sur Def $\sum_{\Sigma}^{m}$. D'autre part le groupe $G L_{h}(\mathcal{O})$ agit de façon naturelle sur les structures de niveau et cela définit des actions de ce groupe sur les différents espaces Def $\Sigma_{\Sigma}^{m}$, actions compatibles aux projections ci-dessus. On voit facilement que la projection de $\operatorname{Def}_{\Sigma}^{m}$ sur Def $0_{\Sigma}^{0}$ constitue un revêtement galoisien de groupe $G L_{h}\left(\mathcal{O} / \varpi^{m} \mathcal{O}\right)$. Ce revêtement est étale en fibre générique, mais au contraire très ramifié au-desssus de la fibre spéciale.

Dans l'article [Ca3] j'avais essayé de généraliser de façon un peu naïve la construction faite dans le cas de la dimension 1 et expliquée dans la première partie de cet exposé : l'idée était de considérer la cohomologie (en degré médian $h-1$ ) de la fibre générique de $\operatorname{Def}_{\Sigma}^{m}$. Toutefois cet espace de cycles évanescents formels ne semble pas avoir en dimension plus grande les bonnes propriétés (analogues au résultat de Brylinski mentionné plus haut) qu'il présente pour les courbes. Un bonne construction des groupes de cycles évanescents dans ce contexte a été fournie par Berkovich (cf. [Be1] [Be2] [Be3]) dans le cadre de sa théorie des espaces analytiques p-adiques. Je renvoie le lecteur intéressé par la théorie de Berkovich à l'exposé de Ducros au séminaire Bourbaki [Du]. C'est pour de toutes autres raisons que Berkovich avait développé cette nouvelle théorie des 
espaces analytiques $p$-adiques, mais elle s'est avérée constituer un cadre parfait pour le développement d'une bonne théorie de la cohomologie étale $\ell$-adique. Par une heureuse coïncidence, il a compris cela précisément au moment où la communauté automorphe avait besoin de ces résultats, ce qui a constitué pour lui un précieux encouragement et un test immédiat; et cela a permis par ailleurs des avancées inespérées du côté automorphe.

On considère donc les espaces de cycles évanescents $\ell$-adiques (où $\ell \neq p$ est un nombre premier fixé) associés par la théorie de Berkovich à la fibre générique de $\operatorname{Def}_{\Sigma}^{m}$ (laquelle est de façon naturelle un espace analytique $p$-adique). Nous noterons ces espaces $\Psi_{K, \ell, h, m}^{i}$ (ou plus simplement $\Psi_{h, m}^{i}$ lorsque $K$ et $\ell$ seront fixés). Lorsque $m$ varie, ils constituent un système inductif (à morphismes injectifs) de limite (réunion) $\Psi_{h}^{i}$.

On a déjà vu que le groupe $G L_{h}(\mathcal{O})$ agissait sur l'espace Def ${ }_{\Sigma}^{m}$ (action sur la structure de niveau). D'autre part, Le groupe $\mathcal{O}_{D_{h}}^{*}$ des éléments inversibles de l'ordre maximal $\mathcal{O}_{D_{h}}$ du corps gauche $D_{h}=D_{K, h}$ est le groupe des automorphismes de $\Sigma_{h}$ et en tant que tel il agit sur l'espace de la déformation universelle et sur $\tilde{\Sigma}_{h}$. Ces deux groupes agissent donc sur les $\Psi_{h, m}^{i}$, et aussi sur leur limite (actions qui commutent entre elles). On a enfin une action du groupe d'inertie $\operatorname{Gal}\left(\bar{K} / K^{\mathrm{nr}}\right)$.

Il est possible (en considérant les isogénies) d'étendre ces actions en une action du sous-groupe :

$$
A_{h} \subset G L_{h}(K) \times D_{h}^{*} \times W_{K}
$$

constitué des triples $(g, d, w)$ qui vérifient la relation : $\operatorname{det}(g)^{-1} N(d) \mathrm{Cl}(w) \in$ $\mathcal{O}^{*}$, où $N$ désigne la norme réduite et $\mathrm{Cl}: W_{K} \rightarrow K^{*}$ l'application de réciprocité de la théorie du corps de classes.

Dans [Ca3] j'avais défini la "représentation locale fondamentale" $\mathcal{U}=\mathcal{U}_{h, K}$ en induisant de $A_{h}$ au produit $G L_{h}(K) \times D_{h}^{*} \times W_{K}$ la représentation $\Psi_{h}^{h-1}$. Pour $\chi$ un caractère (à valeurs dans $\overline{\mathbb{Q}}_{\ell}{ }^{*}$ ) du groupe $K^{*}$, notons $\mathcal{U}(\chi)$ le sous-espace de $\mathcal{U}$ où le centre de $G L_{h}(K)$ agit par $\chi$.

A l'époque où j'avais rédigé cet article les définitions de Berkovich n'étaient pas encore connues et j'avais défini les choses "naïvement" (considérant la cohomologie de la fibre générique, vue algébriquement). De ce fait la conjecture que j'avais énoncée n'était pas correcte, elle le devient "presque" en remplaçant ce point de vue naïf par celui de Berkovich. La conjecture prédisait une décomposition :

$$
\mathcal{U}(\chi)=\bigoplus \pi \otimes j(\pi)^{\vee} \otimes \mathcal{L}(\pi)^{\prime}
$$


où $\pi$ décrit l'ensemble des représentations, de caractère central $\chi$, de la série discrète de $G L_{h}(K)$. Les deux autres constituants du produit tensoriel sont $j(\pi)^{\vee}$, la contragrédiente de la représentation de $D_{h}^{*}$ associée à $\pi$ par la correspondance de Jacquet-Langlands généralisée (connue à l'époque seulement dans le cas des corps $p$-adiques, le cas des corps de fonctions est beaucoup plus récent et dû à Badulescu). $\mathcal{L}$ désigne la correspondance de Langlands locale, convenablement normalisée (entre représentations de $G L_{h}(K)$ et représentations du groupe de Weil, et qui n'était alors établie que pour $h=1,2$ ou 3.) Enfin $\mathcal{L}(\pi)^{\prime}$ désignait un quotient de $\mathcal{L}(\pi)$ (différent de $\mathcal{L}(\pi)$ seulement dans le cas où $\pi$ n'est pas cuspidale).

La conjecture ainsi énoncée est vraie pour $h=1$ : c'est un exercice de vérifier qu'elle ne constitue qu'une reformulation de la théorie de Lubin-Tate. Elle l'est aussi pour $h=2$ sur un corps $p$-adique : c'est ce qu'il résulte de mes travaux issus de la méthode de Deligne et dont j'ai donné une idée dans le paragraphe précédent. Pour $h>2$ la partie de cette conjecture relative aux cuspidales est vraie; autrement dit la partie isotypique correspondant à $\pi\left(\right.$ ou $\left.j(\pi)^{\vee}\right)$ est bien celle que l'on attend : cela a été prouvé par Boyer en 1998 dans le cas des corps d'égale caractéristique puis, peu de temps après, par Harris et Taylor dans un travail où ils prouvaient l'existence de la correspondance de Langlands locale pour les corps $p$-adiques (dont on avait démontré l'existence, en égale caractéristique, dans l'article [LRS] de Laumon, Rapoport et Stuhler). Dans le cas des représentations non cuspidales, les choses sont plus compliquées et ont été élucidées, plus récemment, par Boyer (voir plus bas). Pour obtenir tous ces résultats on utilise de nouveau une comparaison entre le "local" et le "global" qui consiste à exprimer des groupes de cycles évanescents de certaines variétés de Shimura (ou de leurs analogues sur les corps de fonctions) en termes de cette représentation locale fondamentale. Dans le paragraphe suivant je vais dire quelques mots au sujet des variétés de Shimura qui interviennent et je renverrai aux articles originaux, ou bien à mon exposé [Ca4] au séminaire Bourbaki, pour plus de détails.

\subsection{Variétés de Shimura associées à certaines formes de groupes} unitaires. - Les variétés considérées sont celles attachées à des formes de groupes unitaires. Plus précisément, on peut définir un tel groupe $G$ de la façon suivante : partant d'un corps totalement réel $F$, on note $F^{\prime}$ le composé de $F$ avec un corps quadratique imaginaire $E$. On se donne une algèbre à division $B$ de centre $F^{\prime}$ et de degré $n^{2}$, munie d'une involution $*$ de seconde espèce (i.e. qui induit sur $F^{\prime}$ la conjugaison par rapport à $F$ ) et positive. On conjugue cette involution par un élément $\beta$ tel que $\beta^{*}=-\beta$, et l'on obtient ainsi une seconde involution $\sharp$ (autrement dit $\left.x^{\sharp}=\beta x^{*} \beta^{-1}\right)$. 
Le groupe auquel on s'intéresse est le groupe $G$ associé à cette involution $\sharp$ : c'est un groupe réductif défini sur $\mathbb{Q}$ dont l'ensemble des points à valeurs dans une $\mathbb{Q}$-algèbre $R$ est donné par :

$$
G(R)=\left\{g \in B \otimes R ; b b^{\sharp} \in R^{*}\right\}=\left\{g \in B \otimes R ; b \beta b^{\sharp} \in \beta R^{*}\right\}
$$

C'est une forme intérieure du groupe de similitudes unitaires de degré $n$ associé au corps $F$. Il est possible de choisir $\beta$ de telle sorte qu'aux places archimédiennes ce groupe soit le produit d'un groupe de type $(n-1,1)$ et de $(d-1)$ groupes de type $(n, 0)$. Le groupe $G(\mathbb{R})$ opère via le premier facteur sur la boule unité $X$ de $\mathbb{C}^{n-1}$, qui constitue l'espace hermitien symétrique associé.

Dans cette situation, on peut définir les variétés de Shimura associées $\mathrm{Sh}_{U}$, avec $U$ un sous-groupe compact ouvert de $G\left(\mathbf{A}_{f}\right)$. Ce sont des variétés algébriques projectives définies sur le corps $F$, dont l'ensemble des points complexes est donné par :

$$
\mathrm{Sh}_{U}(\mathbb{C})=G(\mathbb{Q}) \backslash\left[X \times G\left(\mathbf{A}_{f}\right) / U\right]
$$

Comme dans le cas des courbes, $\mathrm{Sh}_{U}(\mathbb{C})$ est en fait une réunion de quotients $\Gamma \backslash X$ de $X$ par des groupes de congruence $\Gamma$ de $G(\mathbf{R})$.

D'après Shimura (voir [De2]), $\mathrm{Sh}_{U}$ admet sur le corps $F$ un modèle que l'on peut (à quelques complications techniques près, que je passerai sous silence) décrire en termes d'un problème de modules faisant intervenir des variétés abéliennes $A$ à isogénie près de dimension $d n^{2}$, munies d'une action de $B$, d'une polarisation et d'une structure de niveau, telles que l'involution de Rosati associée induise l'involution $*$ sur $B$; une condition décrivant l'algèbre de Lie de $A$ doit être satisfaite.

Utilisant ce problème de modules, il est possible de décrire la réduction des variétés $\mathrm{Sh}_{U}$ en une place $\mathfrak{P}$ de $F$, de caractéristique résiduelle $p$, telle que le corps $E$ soit décomposé en $p$ et que l'algèbre $B$ soit déployée aux deux places de $F^{\prime}$ qui relèvent $\mathfrak{P}$. On note $\mathfrak{P}_{1}=\mathfrak{P}$, et $\mathfrak{P}_{2} \cdots \mathfrak{P}_{r}$ les autres places de $F$ de caractéristique résiduelle $p$. On les relève en des places $\mathfrak{P}_{i}^{\prime}$ de $F^{\prime}$ (au dessus d'une même place de $E)$. Le groupe $G\left(\mathbb{Q}_{p}\right)$ est isomorphe à $\mathbb{Q}_{p}^{*} \times G L_{n}\left(F_{\mathfrak{P}_{1}}\right) \times$ $B_{\mathfrak{P}_{2}^{\prime}}^{*} \times \cdots \times B_{\mathfrak{P}_{r}^{\prime}}^{*}$. On suppose que le sous-groupe $U$ est décomposé en un produit $U^{p} \times U_{p}$, avec $U^{p} \subset G\left(\mathbf{A}_{f}^{p}\right)$ (adèles finies sans la composante en $p$ ) et que $U_{p} \subset G\left(\mathbf{Q}_{p}\right)$ est lui-même décomposé en le produit du sous-groupe $\mathbf{Z}_{p}^{*}$ de $\mathbf{Q}_{p}^{*}$ et de sous-groupes $U_{\mathfrak{P}_{1}} \subset G L_{n}\left(F_{\mathfrak{P}_{1}}\right)$ et $U_{\mathfrak{P}_{i}^{\prime}} \subset B_{\mathfrak{P}_{i}^{\prime}}^{*}$.

Dans ces conditions, on montre que notre variété a bonne réduction lorsque $U_{\mathfrak{P}_{1}}$ est le sous groupe compact maximal $G L_{n}\left(\mathcal{O}_{F, \mathfrak{P}}\right) \subset G L_{n}\left(F_{\mathfrak{P}}\right)$ tandis que, lorsque $U_{\mathfrak{P}_{1}}$ est un sous-groupe de congruence dans $G L_{n}\left(\mathcal{O}_{F, \mathfrak{P}}\right)$, cette variété présente une mauvaise réduction dont la théorie généralise celle des courbes 
modulaires. Nous noterons encore $\mathrm{Sh}_{U}$ un modèle sur $\mathcal{O}=\mathcal{O}_{F, \mathfrak{P}}$ de notre variété.

La raison fondamentale de cette similitude avec la réduction des courbes modulaires est que toute la théorie de la réduction des variétés que nous considérons est gouvernée par un $\mathcal{O}_{F, \mathfrak{R}}$-module divisible $\mathcal{G}$ qui joue le rôle du groupe $p$ divisible de la courbe elliptique universelle sur les courbes modulaires. C'était d'ailleurs déjà ce fait qui permettait de comprendre la réduction des courbes de Shimura en une place de déploiement de l'algèbre de quaternions.

Donnons une idée approximative de la construction de ce module divisible, qui constitue un morceau du groupe $p$-divisible d'un schéma abélien : le schéma de modules (défini sur $\mathcal{O}=\mathcal{O}_{F, \mathfrak{P}}$ ) associé à la variété de Shimura fait intervenir un schéma abélien $\mathcal{A}$ (qu'on préfère maintenant ne plus considérer à isogénie près) polarisé, muni d'une action d'un ordre $\mathcal{O}_{B} \subset B$; cet ordre doit être stable par l'involution $*$, tel que sa complétion en $p$ soit maximale, et tel que $\mathcal{O}_{B, \mathfrak{P}_{1}}=M_{n}\left(\mathcal{O}_{F, \mathfrak{P}_{1}}\right)=M_{n}(\mathcal{O})$.

On décompose ensuite le groupe $p$-divisible associé à $\mathcal{A}$ sous l'action de l'algèbre $\mathcal{O}_{F} \otimes \mathbb{Z}_{p} \subset \mathcal{O}_{B} \otimes \mathbb{Z}_{p}$. On obtient de la sorte une somme directe faisant apparaître les différentes places $\mathfrak{P}_{i}^{\prime}$ ainsi que leurs conjuguées (par rapport à $F$ ) $\mathfrak{P}_{i}^{\prime \prime}$ :

$$
\mathcal{A}_{p^{\infty}}=\mathcal{A}_{\mathfrak{P}_{1}^{\prime} \infty} \oplus \mathcal{A}_{\mathfrak{P}_{2}^{\prime}} \oplus \cdots \oplus \mathcal{A}_{\mathfrak{P}^{\prime} \infty} \oplus \mathcal{A}_{\mathfrak{P}^{\prime \prime}}{ }_{1}^{\infty} \oplus \mathcal{A}_{\mathfrak{P}^{\prime \prime}}{ }_{2}^{\infty} \oplus \cdots \oplus \mathcal{A}_{\mathfrak{P}^{\prime \prime}} \oplus
$$

Les conditions mentionnées plus haut et portant sur l'algèbre de Lie forcent les groupes $\mathcal{A}_{\mathfrak{P}^{\prime \prime}}{ }_{2} \cdots \mathcal{A}_{\mathfrak{P}^{\prime \prime}}{ }_{r}^{\infty}$ à être ind-étales (ce qui fait que les structures de niveau sont les "mêmes" qu'en caractéristique 0). D'autre part, $\mathcal{A}_{\mathfrak{P}^{\prime \prime}{ }_{1}^{\infty}}$ est un $M_{n}(\mathcal{O})$-module et se décompose de ce fait ("équivalence de Morita") en la somme de $n$ copies d'un $\mathcal{O}$-module noté $\mathcal{G}$. Alors les conditions imposées font que $\mathcal{G}$ est un $\mathcal{O}$-module divisible de dimension infinitésimale 1 et de hauteur totale $n$.

C'est ce groupe qui joue un rôle fondamental. Ses déformations permettent de reconstituer celles de $\mathcal{A}_{\mathfrak{P}^{\prime \prime}}{ }_{1}^{\infty}$ puis de tout $\mathcal{A}_{p}$ (en effet, les déformations des $\mathcal{A}_{\mathfrak{P}^{\prime \prime}}{ }_{i}^{\infty}$ sont triviales pour $i>1$ et l'on a une dualité de Cartier qui pour chaque $i$ lie $\mathcal{A}_{\mathfrak{P}_{i}^{\prime}}$ et $\mathcal{A}_{\mathfrak{P}^{\prime \prime}}{ }_{i}^{\infty}$ ). Ainsi on a un analogue du théorème de SerreTate. Lorsque le groupe $U_{\mathfrak{P}_{1}}$ est un sous groupe de congruence distinct de $G L_{n}(\mathcal{O})$, la mauvaise réduction se décrit en termes de structures (bases de Drinfeld) sur ce module $\mathcal{G}$.

Sur la fibre spéciale $\mathrm{Sh}_{U \mid s}=\mathrm{Sh}_{U} \otimes_{\mathcal{O}} k$ la hauteur de la partie infinitésimale (resp. étale) de $\mathcal{G}$ varie entre 1 et $n$ et cela définit une stratification de cette 
fibre. On notera dans la suite $\mathrm{Sh}_{U}^{[h]}$ le lieu où la hauteur de la partie étale est $\leq h$ (ce sont des fermés, qui constituent une suite croissante) et $\operatorname{Sh}_{U}^{(h)}$ le complémentaire de $\operatorname{Sh}_{U \mid s}^{[h-1]}$ dans $\operatorname{Sh}_{U \mid s}^{[h]}$ (localement fermé de dimension h). En particulier $\mathrm{Sh}_{U \mid s}^{[0]}=\mathrm{Sh}_{U}^{(0)}$ est constitué d'un nombre fini de points "supersinguliers" (tels que le groupe $\mathcal{G}$ soit purement infinitésimal). Les cycles évanescents le long de $\mathrm{Sh}_{U}^{(h)}$ sont liés aux déformations de $\Sigma_{n-h}$ et donc à la représentation locale $\Psi_{n-h}^{i}$. Le lien exact a été élucidé par Harris et Taylor ([HT]) et nous allons dans le paragraphe suivant donner une idée de leurs constructions.

En égale caractéristique $p$, autrement dit sur les corps de fonctions d'une variable sur un corps fini, on a des analogues locaux des variétés de Shimura associés à nos groupes unitaires. On pourrait partir des variétés modulaires de Drinfeld (classifiant des "modules elliptiques") ou bien des Chtoukas. On a plutôt travaillé avec des analogues propres, construits pas Laumon, Rapoport et Stuhler ([LRS]). Ces variétés sont également gouvernées par un $\mathcal{O}$-module divisible.

3.3. Thèse de Boyer et travaux de Harris-Taylor. - La thèse de Pascal Boyer a constitué le premier pas vers une généralisation en dimension supérieure de la méthode de Deligne. Dans ce travail, il se place en égale caractéristique et parvient à démontrer le résultat voulu en généralisant cette méthode au cas des variétés de Deligne-Rapoport-Stuhler. Un point important dans cette thèse consistait à établir un analogue, dans cette situation, du théorème de Serre et Tate. Une autre étape essentielle consistait à montrer que les strates autres que la strate supersingulière ne pouvaient contribuer aux représentations cuspidales de $G L_{n}$ (ce qui généralise des idées contenues dans l'article [PS] de Piatetskii-Shapiro). Avec tout cela il pouvait appliquer une méthode semblable à celle de Deligne : il montrait en gros que la représentation locale fondamentale réalise - du moins dans sa partie reliée aux cuspidales - une correspondance entre représentations des trois groupes $G L_{n}(K), D_{n}^{*}$ et $W_{K}$ qui est la correspondance de Jacquet-Langlands généralisée entre les deux premiers. Par aileurs, elle est telle que la représentation du groupe de Weil ainsi associée à une représentation cuspidale $\pi_{v}$ coïncide avec la restriction au groupe de Weil local d'une représentation galoisienne provenant d'une représentation automorphe $\Pi$ de composante locale $\pi_{v}$. Or pour les corps de fonctions, on sait que les représentations locales automorphes et galoisiennes se correspondent partout par la correspondance de Langlands locale (dont on démontre ainsi 
l'existence [LRS]) : cela est une conséquence du fait que l'on sait que les fonctions $L$ admettent des équations fonctionelles avec des constantes exprimables en termes de constantes locales.

Pour les corps de nombres, la situation paraissait moins favorable car s'il était relativement clair que les travaux de Boyer pouvaient s'étendre à ce cas, on ne connaissait pas l'existence de la correspondance de Langlands locale dans ce cas (ni les bonnes propriétés requises des fonctions $L$ ). A l'époque, j'étais pessimiste sur cette question et ne pensais pas que ces méthodes géométriques, que j'avais pourtant contribué à promouvoir, puissent donner les résultats voulus.

Mais j'avais tort... Que cela soit possible résulte d'une idée, rétrospectivement assez élémentaire, mais fondamentale et qui m'avait complétement échappé. C'est Harris (dans [Har2]) qui a découvert cela. Expliquons, de façon un peu approximative, de quoi il s'agit. Utilisant la représentation locale fondamentale, on associe à chaque représentation cuspidale $\pi$ de $G L_{n}(K)$ une représentation galoisienne $\sigma_{n}(\pi)$. Il est alors possible, en ne considérant que les points supersinguliers des variétés de Shimura discutées au numéro précédent (et asociées à un groupe unitaire en $n$ variables) de montrer que cette application $\sigma$ "calcule" la restriction en une place (où le groupe est déployé) de la représentation galoisienne attachée à une représentation automorphe $\Pi$ de composante cuspidale en cette place.

L'idée de Harris consiste à étendre cette application $\sigma$ aux induites irréductibles de cuspidales. Soit $n$ un entier, dont on se donne une partition $n=n_{1}+n_{2}+$ $\cdots+n_{k}$. Si $\pi_{1}, \cdots \pi_{k}$ sont des représentations cuspidales des groupes $G L_{n_{i}}(K)$, on note $\pi=\pi_{1} \times \pi_{2} \times \cdots \times \pi_{k}$ l'induite (induction unitaire) à partir du parabolique de $G L_{n}(K)$, associé à la partition considérée, de la représentation $\pi_{1} \otimes \pi_{2} \otimes \cdots \otimes \pi_{k}$ (une représentation du sous-groupe de Levi que l'on étend trivialement). On suppose que cette induite est irréductible. Alors on lui associe la représentation galoisienne :

$$
\tilde{\sigma}_{n}(\pi)=\sigma_{n_{1}}\left(\pi_{1}\right) \otimes||^{n_{1}-n} \bigoplus \sigma_{n_{2}}\left(\pi_{2}\right) \otimes||^{n_{2}-n} \bigoplus \cdots \bigoplus \sigma_{n_{k}}\left(\pi_{k}\right) \otimes||^{n_{k}-n}
$$

Supposons qu'il soit encore vrai que cette application "calcule" (à semisimplification près) la restriction en une place de la représentation galoisienne attachée à une représentation automorphe $\Pi$ de composante $\pi$, non nécessairement cuspidale. Alors les applications $\sigma_{h}$ vérifient bien les conjectures de Langlands locales (une propriété qui signifie que des facteurs epsilon, que l'on sait définir tant du côté automorphe que galoisien, sont préservés). Harris a découvert que cette propriété était une conséquence du théorème d'induction de Brauer, des propriétés des facteurs $\epsilon$ locaux et de l'existence de l'induction automorphe et de la construction des représentations galoisiennes attachées aux formes automorphes. 
Le cœur du travail de Harris et Taylor consiste a prouver cette propriété et cela nécessite de comprendre non pas seulement les cycles évanescents aux points supersinguliers du schéma $\mathrm{Sh}_{U}$ mais aussi le long des différentes strates $\mathrm{Sh}_{U}^{(h)}$. La structure de ce schéma le long d'une telle strate est contrôlée par les déformations du $\mathcal{O}$-module divisible $\mathcal{G}$, dont la partie étale $\mathcal{G}^{\text {et }}$ est localement isomorphe à $\left(\varpi^{-m} \mathcal{O} / \mathcal{O}\right)^{h}$ et la partie infinitésimale $\mathcal{G}^{0}$ à $\Sigma_{n-h}$.

Supposons, comme au paragraphe précédent, que le sous-groupe $U$ est décomposé en un produit de sous-groupes relatifs aux différentes places de $F$. On écrit $U=U^{\mathfrak{P}} \times U_{\mathfrak{P}}$, où $U_{\mathfrak{P}}$ désigne la composante en $\mathfrak{P}$ et $U^{\mathfrak{P}}$ le produit de toutes les autres. On suppose de plus que $U_{\mathfrak{P}}=U_{\mathfrak{P}}^{m}$ coïncide avec le $m$ ième groupe de congruence dans $G L_{n}\left(\mathcal{O}_{F, \mathfrak{P}}\right)$. On note alors $\mathrm{Sh}_{m, U_{\mathfrak{P}}}$ la variété correspondante (ainsi qu'un modèle sur l'anneau $\mathcal{O}$ ). Un tel modèle est lisse lorsque $m=0$ et devient ramifié dès que $m>0$.

Pour comprendre les cycles évanescents le long des différentes strates, on introduit les variétés d'Igusa (généralisant les courbes du même nom qui interviennent dans la théorie des courbes modulaires). Ce sont des variétés sur le corps résiduel $k$. Les variétés d'Igusa de première espèce sont les revêtements, notés $I_{U^{\mathfrak{P}}, m}^{(h)}$, de la partie $\operatorname{Sh}_{m, U^{\mathfrak{P}} \mid s}{ }_{s}(h)$ de la fibre spéciale où $\mathcal{G}^{\text {et }}$ est de hauteur $h$. Un tel revêtement est défini comme classifiant les isomorphismes (structures de niveau partielles) entre $\left(\varpi^{-m} \mathcal{O} / \mathcal{O}\right)^{h}$ et $(\mathcal{G})_{\varpi m}^{\text {et }}$. Il est étale galoisien de groupe $G L_{h}\left(\mathcal{O} / \varpi^{m} \mathcal{O}\right)$.

La variété d'Igusa $I_{U^{\mathfrak{P}}, m}^{(h)}$ est la fibre spéciale d'un schéma formel $I_{U^{\mathfrak{N}}, m}^{(h) \wedge}$, classifiant les structures de niveau étales partielles comme plus haut, au dessus du complété de $\mathrm{Sh}_{0, U^{\mathfrak{Y}}}$ le long de sa fibre spéciale $\mathrm{Sh}_{0, U^{\mathfrak{P}}}{ }_{\mid s}(h)$. On peut définir au-dessus de $I_{U^{\mathfrak{\gamma}}, m}^{(h)}$ un revêtement noté $I_{U^{\mathfrak{N}}, m}^{(h) \wedge}(t)$ (cette fois-ci très ramifié au dessus de la fibre spéciale) qui classifie les bases de Drinfeld partielles :

$$
\left(\varpi^{-t} \mathcal{O} / \mathcal{O}\right)^{n-h} \rightarrow(\mathcal{G})_{\varpi^{t}}^{0}
$$

vers la partie formelle $(\mathcal{G})_{\varpi^{t}}^{0}$ de $(\mathcal{G})_{\varpi^{t}}$. Ce revêtement constitue un modèle formel de $\mathrm{Sh}_{m, U^{\mathfrak{P}}}$ au voisinage de sa fibre spéciale et la théorie de Berkovich nous permet alors d'identifier les espaces cycles évanescents que nous voulons calculer à ceux de $I_{U^{\mathfrak{x}}, m}^{(h) \wedge}(m)$.

Notons $\Phi^{j}(t)$ ce faisceau de cycles évanescents (au-dessus de $I_{U^{\mathfrak{x}}, m}^{(h)}$ ). Il s'agit d'exprimer ce faisceau en termes de la représentation locale fondamentale. En un $\bar{k}$-point $x$ de la fibre spéciale, l'espace des déformations de $\mathcal{G}_{x}$ se projette sur celui des déformations de la partie formelle $\mathcal{G}_{x}^{0}$ et on vérifie sans peine que 
cette projection est formellement lisse. Si l'on fixe un isomorphisme entre $\mathcal{G}_{x}^{0}$ et $\Sigma_{n-h}$, on obtient alors un isomorphisme entre les espaces correspondants de déformations et donc entre les espaces de cyles évanescents $\Phi^{j}(t)$ et $\Psi_{n-h, t}^{j}$. Mais le groupe $\mathcal{G}^{0}$ n'est pas constant et il n'existe donc pas de tel isomorphisme sur $I_{U^{\mathfrak{x}}, m}^{(h)}$. Pour remédier à cela, on introduit les variétés d'Igusa de seconde espèce :

$$
J_{U^{\mathcal{P}}, m, s}^{(h)} \longrightarrow I_{U^{\mathcal{P}}, m}^{(h)} \otimes_{k} \bar{k}
$$

qui classifient les isomorphismes entre les sous-groupes de points de $\varpi^{s}$-torsion du groupe $\Sigma_{n-h}$ et du groupe $\mathcal{G}^{0}$. Ces revêtements constituent (pour s variant) un système projectif de revêtements étales de $I_{U^{w}, m}^{(h)} \otimes_{k} \bar{k}$, de groupe de Galois $\mathcal{O}_{D_{n-h}}^{*}$. Intuitivement ce système trivialise notre groupe $\mathcal{G}^{0}$ et donc le faisceau des cycles évanescents, qui devient sur la limite isomorphe à $\Psi_{n-h, t}^{j}$. Utilisant la théorie de Berkovich et un passage à la limite (un peu délicat) on peut effectivement montrer que le faisceau des cycles évanescents sur $I_{U^{\mathcal{P}}, m}^{(h)} \otimes_{k} \bar{k}$ est celui obtenu à partir de faisceau constant $\Psi_{n-h, t}^{j}$ sur la limite projective $\operatorname{des} J_{U^{\mathcal{P}}, m, s}^{(h)}$ par passage au quotient par $\mathcal{O}_{D_{n-h}}^{*}$ (lequel opère, rappelons-le, sur la représentation locale). Cela peut s'exprimer autrement, en considérant l'ensemble des représentations (admissibles, irréductibles) $\rho$ de $D_{n-h}^{*}$ (modulo torsion par un caracère non ramifié). Chacune définit (à partir du système projectif des variétés d'Igusa de seconde espèce) un système local $\mathcal{F}(\rho)$ sur $I_{U^{\mathfrak{R}}, m}^{(h)}$. On obtient alors un isomorphisme :

$$
\Phi^{j}(t)=\bigoplus_{\rho} \mathcal{F}(\rho) \otimes \Psi_{n-h, t}^{j}(\rho) .
$$

Telle est la relation entre les cycles évanescents et la représentation locale. Harris et Taylor utilisent ensuite une méthode englobant à la fois celle de Deligne dont nous avons expliqué le principe dans la première partie, et celle initiée par Langlands et qui consiste à comparer les formules des traces de Selberg et de Lefschetz. On doit pour cela décrire l'ensemble des points des variétés d'Igusa, munies des actions des différents groupes qui interviennent. Le résultat obtenu finalement -avec une extrême virtuosité- prouve finalement que la restriction au groupe de Galois local en $\mathfrak{P}$ peut se calculer de façon purement locale, et c'est ce que l'on voulait. On peut alors conclure en appliquant le résultat de Harris expliqué ci-dessus.

Énonçons le résultat qu'ils obtiennent. On part d'une représentation automorphe $\Pi$ qui apparaît dans la cohomologie (à valeurs dans un faisceau) de notre variété de Shimura. On note $R(\Pi)$ la représentation galoisienne correspondante; on ne fixe pas le degré de cohomologie mais on considère plutôt 
la représentation virtuelle $(-1)^{n-1} \sum_{i}(-1)^{i}\left[R^{i}(\Pi)\right]$. Supposons que la composante locale en $\mathfrak{P}$ est une induite $\Pi_{\mathfrak{p}}=\pi=\pi_{1} \times \pi_{2} \times \cdots \times \pi_{t}$ de cuspidales. Alors la représentation (virtuelle) $[R(\Pi)]$, restreinte au groupe de Weil local en $\mathfrak{p}$ (ce qu'on note par un indice $\mathfrak{p}$ ), est donnée par la formule :

$$
n\left[R(\Pi)_{\mathfrak{p}}\right]=\operatorname{dim}([R(\Pi)])\left[\tilde{\sigma}_{n}\left(\Pi_{\mathfrak{p}}\right)\right] .
$$

3.4. Autres travaux, et résultats plus récents. - Dans cet exposé, je me suis attaché à un aspect particulier de cette théorie, qui consiste en l'étude des cycles évanescents pour des variétés de Shimura associées à des groupes unitaires d'un certain type, en une place où le groupe est déployé (isomorphe à $\left.G L_{n}(K)\right)$. On a des résultats semblables en une place où le groupe est isomorphe au groupe multiplicatif du corps gauche $D_{K, n}^{*}$ de centre $K$ de dimension $n^{2}$ et d'invariant $1 / n$. Les espaces qui interviennent sont alors des espaces analytiques $p$-adiques : le demi-plan généralisé de Drinfeld $\Omega_{K}^{n}$ consiste en l'espace projectif de dimension $n-1$ privé de tous ses hyperplans rationnels, et Drinfeld ([Dr2]) en a construit une famille de revêtements $\operatorname{Rev}_{K}^{n, m}$. La cohomologie de ces objets fournit encore des représentations du produit $G L_{n}(K) \times D_{n}^{*} \times W_{K}$. On obtient ainsi une seconde représentation locale fondamentale et on montre qu'elle possède exactement les mêmes propriétés que celle décrite ici : pour les corps de fonctions cela résulte du travail de Thomas Hausberger [Hau] tandis que dans les cas des corps $p$-adiques cela résulte de [Har1] et [HT]. Cela avait été conjecturé (du moins implicitement) par Drinfeld dès le milieu des années 70 .

La relation entre ces deux types d'objets géométriques, qui produisent au bout du compte des représentations identiques, a été pendant longtemps une question ouverte. Ceux dont nous avons parlé plus haut constituent un système de revêtements, de groupe $G L_{n}\left(\mathcal{O}_{K}\right)$, d'une base où opère $\mathcal{O}_{D_{n}^{*}}$. Pour ceux du type Drinfeld, les groupes jouent des rôles symétriques, i.e. c'est $G L_{n}(K)$ qui opère sur $\Omega_{K}^{n}$ (action évidente) tandis que les revêtements admettent pour groupes de Galois des quotients de $\mathcal{O}_{D_{n}^{*}}$.

Ce n'est qu'assez récemment que Faltings [Fal] (voir aussi [FGL]) est parvenu à prouver que les limites projectives des deux systèmes (vues dans une catégorie convenable) coïncidaient.

La méthode de Harris et Taylor, fondée sur des calculs de formules de traces, ne déterminait que des représentations virtuelles, sommes alternées des espaces correspondant aux différents degrés de cohomologie. Cela permet d'obtenir essentiellement la partie des représentations (locales et globales) correspondant 
aux cuspidales. Boyer ([Boy2], [Boy3]) est finalement venu a bout de la description de la filtration de monodromie dans l'étude des suites spectrales correspondantes, et cela lui permet une description complète des différents objets tant locaux que globaux.

Signalons également un article de Taylor et Yoshida [TY], dans lequel ils déterminent la monodromie des représentations galoisiennes qui apparaïssent en cohomologie de degré médian (par une méthode plus simple que celle de Boyer, mais qui ne va pas aussi loin).

Je mentionnerai aussi le travail [Da] de Dat, dans lequel il considère les mêmes objets que ci-dessus, mais se plaçant dans la catégorie dérivée des représentations lisses de $G L_{n}(K)$, et il obtient des résultats plus fins, pour les représentations non cuspidales mais "elliptiques", qu'en considérant simplement les groupes de cohomologie.

Je renvoie à l'exposé de Thomas Hausberger pour une description plus approfondie de ces points, en particulier des travaux de Boyer et de ceux de Dat.

Je terminerai cet exposé en disant quelques mots de ce qu'il advient lorsqu'on sort du cadre des formes de $G L_{n}$. Rapoport et Zink [RZ2] ont construit des espaces analytiques qui généralisent ceux considérés dans cet exposé, et qui permettent d'uniformiser les différentes "strates" de la fibre spéciale des variétés de Shimura de type PEL (ou même EL). Utilisant les modèles locaux ainsi obtenus, on peut espérer calculer la cohomologie des variétés de Shimura en une place de mauvaise réduction, ce que l'on sait faire dans certains cas (même si la théorie est moins avancée pour de tels groupes généraux). Il s'agit pour l'instant essentiellement de la mauvaise réduction de type parahorique. Cette théorie a connu ces dernières années de multiples et importants développements. Je renverrai le lecteur intéressé par ces modèles locaux à l'article d'exposition [Ra]. L. Fargues ([Far]) et E. Mantovan ([Ma]) ont utilisé les espaces de Rapoport-Zink pour étendre le travail de Harris et Taylor à des groupes unitaires d'un type plus général que ceux considérés dans [HT]. Voir aussi [RZ1] pour des calculs de cycles évanescents dans un esprit un peu diférent de ce que nous avons exposé ici.

\section{Références}

[Be1] V.G. Berkovich, étale cohomology for non-archimedean analytic spaces, Publ. Math. I.H.E.S. 78 (1993), 5-161.

[Be2] V.G. Berkovich, Vanishing cycles for formal schemes, Invent. Math. 115 (1994), $539-571$.

[Be3] V.G. Berkovich, Vanishing cycles for formal schemes II, Invent. Math. 125 (1996), 367-390.

[Boy1] P. Boyer, Mauvaise réduction des variétés de Drinfeld et conjecture de Langlands locale, Invent. Math. 138 (1999) 573-629. 
[Boy2] P. Boyer, Faisceau pervers des cycles évanescents des variétés de Drinfeld et filtration de monodromie locale du modèle de Deligne-Carayol, à paraître aux Mémoires de la SMF.

[Boy3] P. Boyer, Monodromie du faisceau pervers des cycles évanescents de quelques variétés de Shimura simples, livre en préparation.

[Br] J. Brylinski, Un lemme sur les cycles évanescents en dimension relative 1, Appendice à [Ca2].

[Ca1] H. Carayol Sur la mauvaise réduction des courbes de Shimura, Compositio Math. 59, 1986, 151-230.

[Ca2] H. Carayol Sur les représentations $\ell$-adiques associées aux formes modulaires de Hilbert, Ann. Sci. E.N.S. 19, 1986, 409-468.

[Ca3] H. Carayol, Non-abelian Lubin-Tate theory, dans "Automorphic forms, Shimura varieties, and L-functions" vol II (L. Clozel et J.S. Milne, eds), Academic Press (1990).

[Ca4] H. Carayol, Preuve de la conjecture de Langlands locale pour $G L_{n}$ : travaux de Harris-Taylor et Henniart, Séminaire Bourbaki. Vol. 1998/1999. Astérisque 266 (2000).

[Da] J.-F. Dat Théorie de Lubin-Tate non abélienne et représentations elliptiques, Invent. Math. 169 (1) (2007) , 75-152.

[De1] P. Deligne Formes modulaires et représentations $\ell$-adiques, Séminaire Bourbaki, exp. 355 (Février 1969). Lectures Notes in Math. 179, 139-172, Springer 1971.

[De2] P. Deligne Travaux de Shimura, Séminaire Bourbaki, exp. 389 (Février 1971). Lectures Notes in Math. 244, 123-165, Springer 1971.

[De3] P. Deligne Le formalisme des cycles évanescents, Groupes de Monodromie en Géométrie Algébrique (SGA7 II) Lectures Notes in Math. 340, 82-115, Springer 1973.

[De4] P. Deligne Lettre à Piatetskii-Shapiro. (1973)

[Dr1] V.G. Drinfeld Elliptic modules, Mat. Sbornik 94 (136) (1994) 594-627.

[Dr2] V.G. Drinfeld Coverings of p-adic symmetric domains, Funkcional. Anal. i Priložen. 10 (2) (1976) 29-40.

[Du] A. Ducros Espaces analytiques p-adiques au sens de Berkovich, Séminaire Bourbaki. Vol. 2005/2006. Astérisque 311 (2007).

[Fal] G. Faltings A relation between two moduli spaces studied by V.G. Drinfeld, Algebraic number theory and algebraic geometry, 115-129, Contemp. Math. 300, Amer. Math. Soc., Providence, RI, 2002.

[Far] L. Fargues Cohomologie des espaces de modules de groupes p-divisibles et correspondance de Langlands locales, Variétés de Shimura, espaces de Rapoport-Zink et correspondances de Langlands locales, Astérisque 291 (2004), 1-199.

[FGL] L. Fargues, A. Genestier, V. Laforgue L'isomorphisme entre les tours de Lubintate et de Drinfeld, Progress in Mathematics Vol. 262, Birkhäuser 2008.

[Har1] M. Harris, Supercuspidal representations in the cohomology of Drinfeld upper half spaces : elaboration of Carayol's program, Invent. Math. 129 (1997), 75-120.

[Har2] M. Harris, The local Langlands conjecture for $G L(n)$ over a $p$-adic field, $n<p$, Invent. Math. 134 (1998), 177-210. 
[Hau] T. Hausberger Uniformisation des variétés de Laumon-Rapoport-Stuhler et conjecture de Drinfeld-Carayol, Ann. Inst. Fourier (Grenoble) 55 (4) (2005) 12851371.

[HT] M. Harris, R. Taylor On the geometry and cohomology of some simple Shimura varieties, Annals of Math. Studies 151, Princeton University Press (2001).

[KM] N. Katz and B. Mazur Arithmetic moduli of elliptic curves, Annals of Math. Studies 108, Princeton University Press (1985).

[Ku] Ph. Kutzko The local langlands conjecture for GL(2), Annals of Math. 112 (1980), 381-412.

[La] R. P. Langlands Modular forms and $\ell$-adic representations, Modular functions of one variable (Antwerp, 1972), Lectures Notes in Math. 349, 361-500, Springer 1973.

[LRS] G. Laumon, M. Rapoport et U. Stuhler, D-elliptic sheaves and the Langlands correspondence, Invent. Math. 113 (1993), 217-338.

[Ma] E. Mantovan On certain unitary groups Shimura varieties, Variétés de Shimura, espaces de Rapoport-Zink et correspondances de Langlands locales, Astérisque 291 (2004), 201-331.

[Ny] L. Nyssen Représentations extraordinaires, Compositio Math. 115, 3 (1999), $329-357$.

[PS] I.I. Piatetskii-Shapiro Zeta functions of modular curves, Modular functions of one variable (Antwerp, 1972), Lectures Notes in Math. 349, 317-360, Springer 1973.

[Ra] M. Rapoport A guide to the reduction modulo $p$ of Shimura varieties, automorphic forms I, astérisque 298 (2005), 271-318.

[Ro] J. Rogawski, Representations of $G L(n)$ and division algebras over a p-adic field, Duke Math. J. 50 (1983), 161-196.

[RZ1] M. Rapoport et T. Zink, Über die lokale Zetafunktion von Shimuravarietäten. Mondromiefiltration und verschwindende Zyklen in ungleicher charakteristik Invent. Math. 68 (1982) nї£i 1, 21-101.

[RZ2] M. Rapoport et T. Zink, Period spaces for p-divisible groups, Annals of Math. Studies 141, Princeton University Press (1996).

[TY] R. Taylor, T. Yoshida, Compatibility of local and global langlands correpondences, J. Amer. math. Soc. 20 (2) (2007) 467-493.

8 janvier 2009

Henri Carayol, Université de Strasbourg et CNRS - 7 Rue René Descartes - F-67084

Strasbourg Cedex - FRANCE 